\title{
Perspectives on metaphyseal conservative stems
}

\author{
F. Falez • F. Casella - G. Panegrossi - F. Favetti - C. Barresi
}

Received: 6 April 2007 / Accepted: 8 October 2007 / Published online: 17 March 2008

\begin{abstract}
Total hip replacement is showing, during the last decades, a progressive evolution toward principles of reduced bone and soft tissue aggression. These principles have become the basis of a new philosophy, tissue sparing surgery. Regarding hip implants, new conservative components have been proposed and developed as an alternative to conventional stems. Technical and biomechanical characteristics of metaphyseal bone-stock-preserving stems are analyzed on the basis of the available literature and our personal experience. Mayo, Nanos and Metha stems represent, under certain aspects, a design evolution starting from shared concepts: reduced femoral violation, non-anatomic geometry, proximal calcar loading and lateral alignment. However, consistent differences are level of neck preservation, cross-sectional geometry and surface finishing. The Mayo component is the most time-tested component and, in our hands, it showed an excellent survivorship at the mid-term follow-up, with an extremely reduced incidence of aseptic loosening (partially reduced by the association with last generation acetabular couplings). For 160 implants followed for a mean of 4.7 years, survivorship was $97.5 \%$ with 4 failed implants: one fracture with unstable stem, 1 septic loosening and 2 aseptic mobilizations. DEXA analysis, performed on 15 cases, showed a good calcar loading and stimulation, but there was significant lateral load transfer to R3-R4 zones, giv-
\end{abstract}

F. Falez (两) · F. Casella · G. Panegrossi · F. Favetti · C. Barresi Department of Orthopaedics and Traumatology

S. Spirito in Sassia Hospital

Largo Tevere in Sassia 1, 00100 Rome, Italy

e-mail: f.falez@flashnet.it ing to the distal part of the stem a function not simply limited to alignment. Metaphyseal conservative stems demonstrated a wide applicability with an essential surgical technique. Moreover, they offer the options of a "conservative revision" with a conventional primary component in case of failure and a "conservative revision" for failed resurfacing implants.

Keywords Arthroplasty · Conservative · Hip · Metaphyseal · DEXA

\section{Introduction}

Total hip replacement is one of the most popular surgical procedures, with a worldwide successful application related to its high standardized outcome. Nevertheless, the clinical and functional results of prostheses have shown, during the last decades, a significant improvement that has gradually pushed their performances beyond their previous limits.

This continuing evolution has progressively modified the morphology, materials, surface finishing and tribologic coupling of the implants, while the widening of indications to increasingly younger patients has led clinicians to experiment with conservative options that preserve as much bone stock as possible.

This last point has become part of a new philosophy, inspired by principles of conservation (both related to surgical approaches and prosthetic implants), called Tissue Sparing Surgery [1]. In particular, bone retaining is based upon the question: why sacrifice an intact anatomic structure, if it can be useful to implant stability [2, 3], joint biomechanics or a future revision procedure? Besides, this statement, giving an implicit contraindication in main- 
taining altered anatomic structures (both congenital and acquired), suggests by itself what could be a proper use of conservative options.

\section{Rationale and design of metaphyseal stems}

Excluding resurfacing implants, which cannot be considered stems due to their particular characteristics, there are two major groups of conservative implants: neck-retaining and metaphyseal bone-stock-preserving stems. The last group, object of this paper, was developed after the understanding of mechanical properties and role of cortical neck and metaphyseal cancellous bone in stress transfer and implant stabilization (primary and biologic fixation) [3].

In 1993, Jasty et al. [4] noted, in an experimental study, that the diaphyseal tract of a traditional femoral component was no longer necessary to implant stability once a proximal fixation was achieved. A few years later, Whiteside et al. [5] tested in vitro different levels of femoral neck preservation and their ability to neutralize torsional forces of femoral components: preservation of $50 \%$ or more of cortical neck was able to produce acceptable micromotions, while $15 \%$ or less produced macromotion with conventional designs.

All these biomechanical considerations led to the birth, in 1984, of a new femoral solution, the Mayo stem [6], (Zimmer, Warsaw, USA), which has been modified over the following years to the actual component. This prosthetic implant is described as an ultrashort, straight and multitapered component that, according to its developer B.F. Morrey, does not need to look like the femur [6]. The Mayo stem has a proximal wide trapezoidal cross-section functional to search a multipoint contact within cortical femoral bone. This innovative geometry is able to stabilize the implant and to preserve proximal cancellous bone (impacted within a cortical shell, to increase torsional and axial stability). For these reasons, an appropriate level of neck osteotomy, able to leave an intact cortical ring in which cancellous bone could be impacted, is mandatory (Fig. 1).

The Mayo stem represents an evolution of principle, already time-tested for conventional straight stems (and recently confirmed by Swanson [8]) of "fit without fill", with necessary modification of proximal shape and fixation to achieve torsional and axial stability. In fact, the extremely reduced diaphyseal segment of this implant is functional just to varus-valgus alignment along the lateral femoral cortex, leaving the diaphyseal canal practically virgin (Fig. 2).

On the basis of the good clinical behavior (mid- and long-term follow-up) of this component, two successive

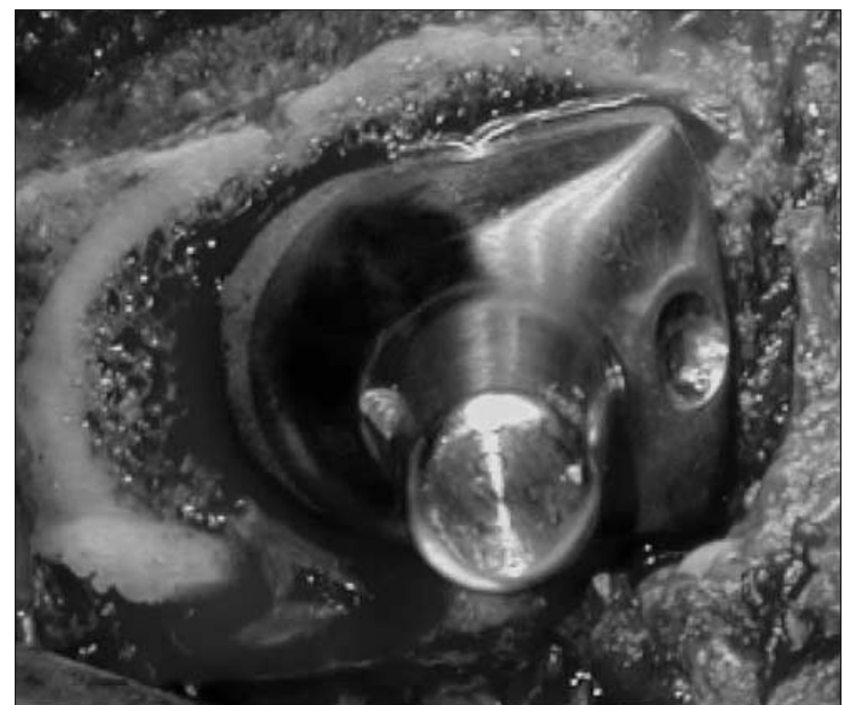

Fig. 1 Intact cortical ring in which cancellous bone can be impacted

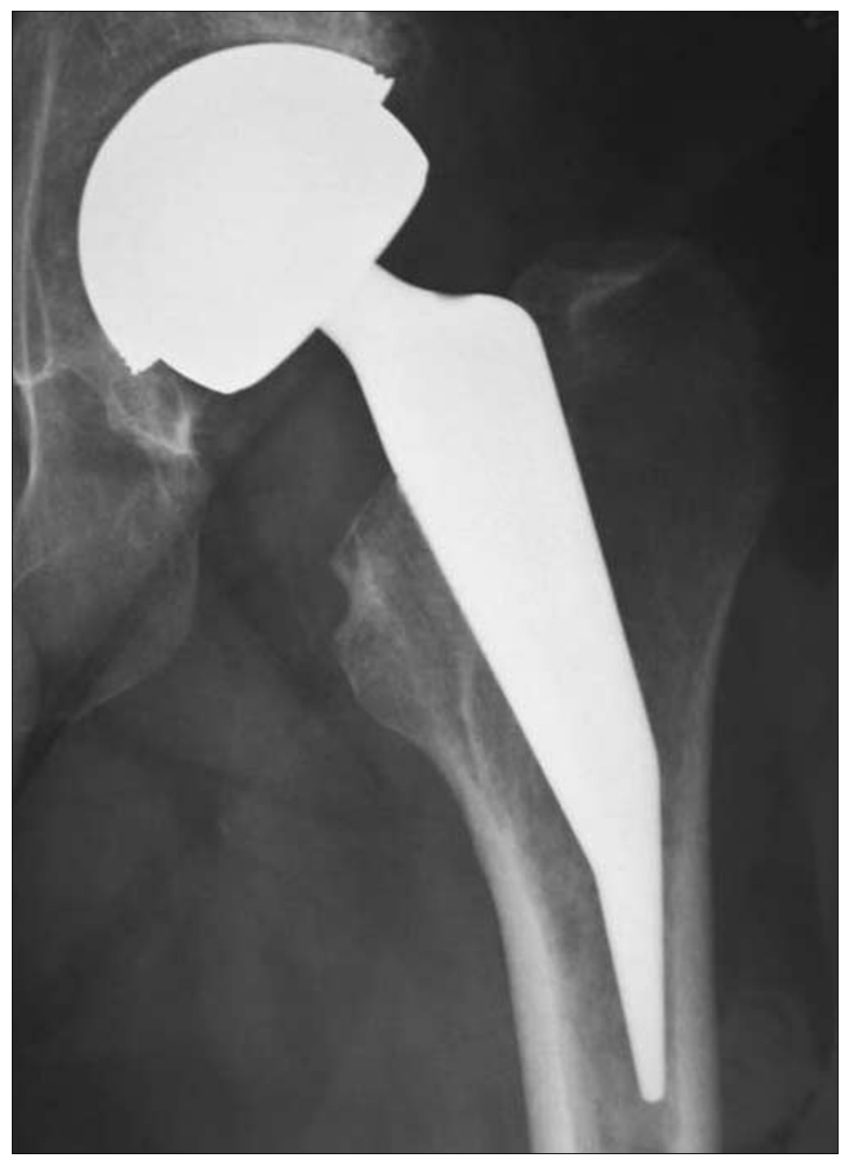

Fig. 2 Mayo reduced diaphyseal segment functional just to varus-valgus alignment along lateral femoral cortex

implants, partially with the same principles, have been introduced: the Nanos stem (Plus Orthopedics, Aarau, Switzerland) and the Metha stem (Bbraun, Melsungen, Germany). They grossly reproduce the shape of the Mayo stem but introduce some variations, the most notable of which is a major neck preservation (which has led to clas- 


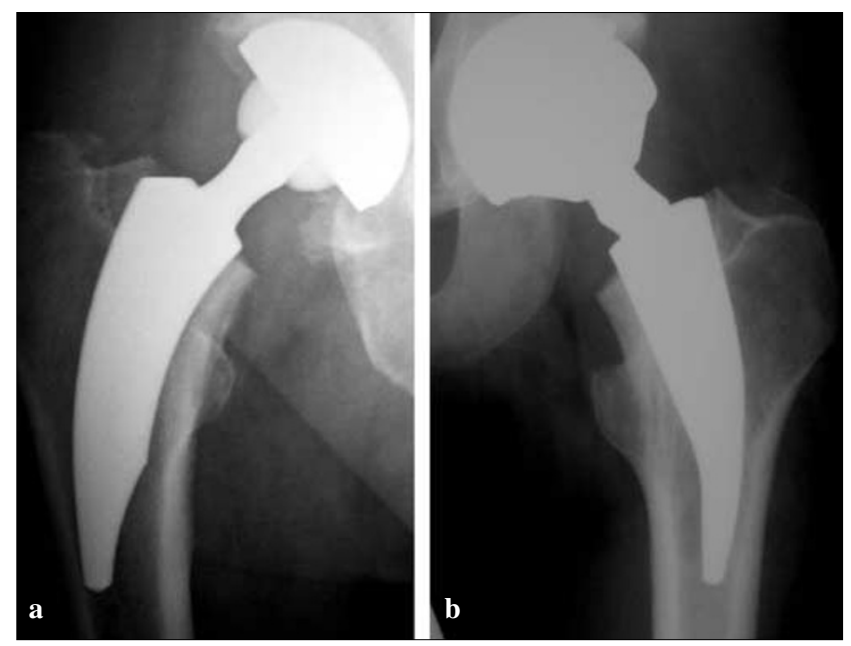

Fig. 3a Nanos stem. b Metha stem

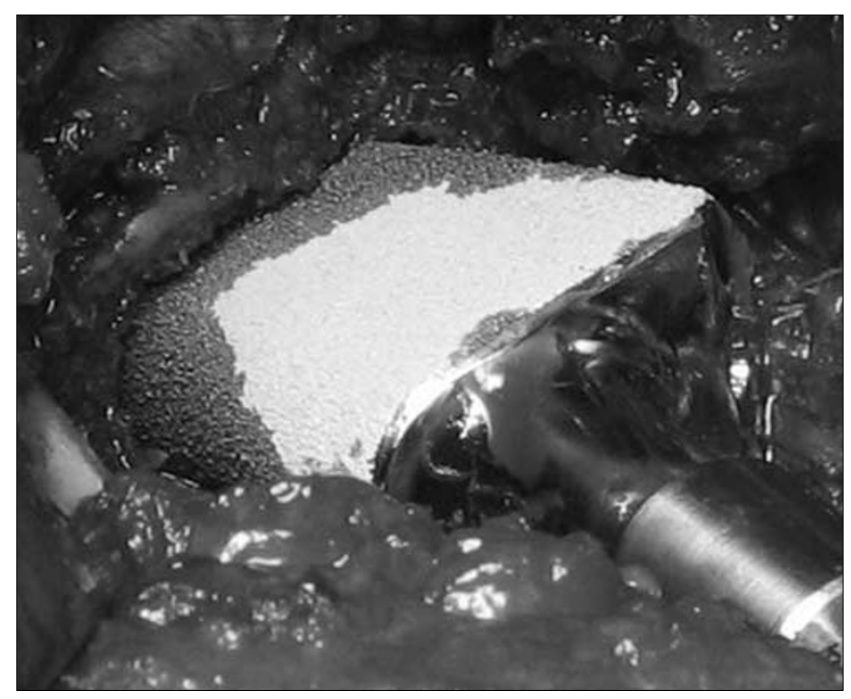

Fig. 4 More proximal filling of Nanos stem (related to its rounded cross-section)

sify these implants within the neck-retaining group) (Fig. 3). A straight and short geometry is common to all these implant, while the Nanos stem presents a rounded crosssection for most of its perimeter and the Metha maintains a trapezoidal section.

These apparently limited variations involve, nevertheless, some differences in technical execution and fixation principles. The Nanos stem requires a higher neck osteotomy and more proximal filling to achieve, with a rounded cross-section, a primary stability, both axial and torsional: this last aspect apparently reduces the validity of proximal cancellous bone preservation (Fig. 4). The Metha stem presents an overall reduction of dimensions (length, cross-section area) compared to the Mayo stem, increasing the amount of retained bone but, on the other hand, requiring an extremely accurate reproduction of neck osteotomy to a level able to allow a proper spongy bone impaction and torsional stability.

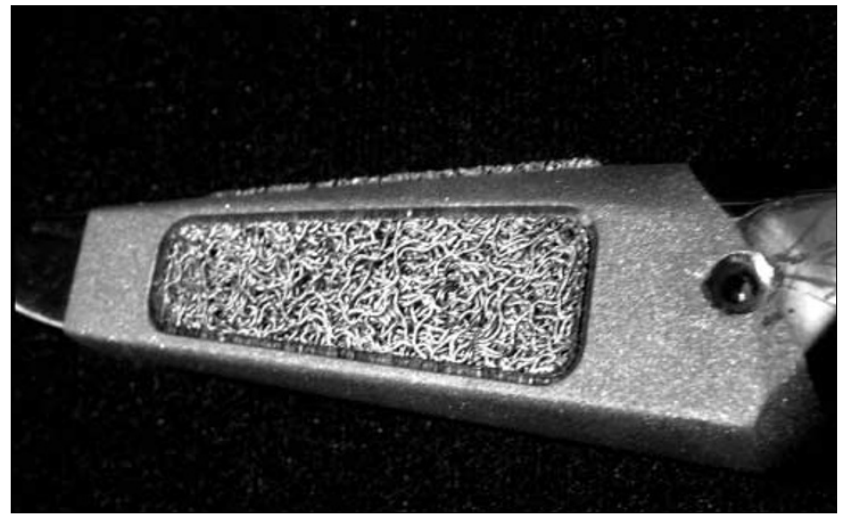

Fig. 5 Surface finishing of Mayo stem: grit blasting with mesh pads

Both these implants offer, doubtless, an evolution in surface finishing: a circumferential, last generation osteoinductive coating replaces the grit-blasting with mesh pads offered by the Mayo stem (Fig. 5), criticized for a potential risk in creating sleeves for distal debris migration (on our advice, initially developed to ensure the maximum mechanical stability at the corner of the trapezoidal cross-section, leaving to the mesh pads the role of secondary bone integration). However, an analysis of a single, explanted, radiographically stable Mayo stem did not detect significant differences in bone integration rate, compared to a conventional cementless implant [9].

\section{Personal experience}

Our experience started in 1999 with the Mayo stem (160 implants, with follow-up ranging from 1 to 7 years) and recently extended to Nanos (2005-2006, 25 implants) and Metha (2006, 27 implants).

Analysis of survivorship, clinical results and complications can be performed only for the most time-tested Mayo stem, but we will also share our preliminary data for the Nanos and Metha stems.

The survivorship of the 160 Mayo stems after a mean of 4.7 years (range, 1-7 years), was $97.5 \%$. Mean age of patients at the implantation was 63.4 years (range, 42-83 years). Main diagnosis was primary arthritis (129 cases, $80.6 \%$ ), osteonecrosis (15 cases, 9.4\%), post-traumatic arthritis (9 cases, 5.6\%), Crowe type I congenital hip dysplasia (CHD; 3 cases, 1.9\%), failed osteosynthesis of proximal femur (2 cases, $1.3 \%$ ) and failed resurfacing arthroplasty ( 2 cases, $1.3 \%$ ).

Four implants failed. There was 1 early postoperative fracture (6 weeks postoperatively) with unstable stem (B2 in Vancouver classification), 1 septic loosening, and 2 aseptic mobilizations after 4 and 5 years. All these 4 implants have been revised. The fracture required a distally stabilized stem (Wagner stem, Zimmer), the septic 
loosening received a two-step revision (antibiotic spacer followed by a primary Versys stem, Zimmer) and the two aseptic mobilizations were revised directly with a conventional primary implant (Platform, Smith\&Nephew, Memphis, USA).

Two more stems are actually closely watched over because of an intra-operative perforation at the tip of the implant, but they show to be clinically and radiographically stable after 3 and 5 years. Other complications detected were 3 intra-operative proximal femoral cracks and 1 intra-operative diaphyseal crack (the last related mainly to a minimally invasive Watson-Jones procedure, with improper femoral manipulation); all were treated with metallic cerclage and not further operated.

One case presented with superolateral thigh pain (a complication usually not detected with this implant [7]) that was related to a cortical stem tip impingment (seen radiographically and with Tc99 scintigraphy). This resolved with pulsed electromagnetic field (PEMF) treatment for 1 month.

A DEXA analysis of 15 of these patients 3 years after implantation, performed as part of a comparative study between different femoral designs [10], showed a good calcar stimulation but a prevalence of distal stress transfer along the stem to the lateral femoral cortex and tip of the stem (R3-R4) giving to the distal segment of the component a function apparently not limited to alignment (Table 1).

Table 1 Periprosthetic bone mineral density (BMD) data in 7 regions of interest. Values are mean (SD) (Modified from [10])

\begin{tabular}{ll}
\hline Region of interest & BMD $\left(\mathrm{g} / \mathrm{cm}^{2}\right)$ \\
\hline R1 & $0.804(0.171)$ \\
R2 & $1.164(0.240)$ \\
R3 & $1.328(0.436)$ \\
R4 & $1.741(0.123)$ \\
R5 & $1.602(0.258)$ \\
R6 & $1.179(0.350)$ \\
R7 & $0.926(0.187)$
\end{tabular}

This result agrees with the radiological appearances of some cases of lateral cortex hipertrophy (however not constant and apparently not related to surgical variables such as under-sizing of the component in the proximal femur) and rare cases of trochanteric stress-shielding (R1R2) (Fig. 6). A widening of the study to a larger sample and a further clinical and radiographic investigation may produce more definitive results on the biotrophism of this femoral component. About this point, another comparative study [11] found that the shortest component tested (Mayo) out of four implant design showed the lowest changes in bone density (however they were again summarized as calcar resorption and distal hipertrophy).

Encouraging results obtained at short- to mid-term follow-up, such as a low failure rate $(2.5 \%)$ and a low specific complication rate $(4.3 \%)$, and the wide versatility to different joint reconstruction indications (practically only mild to severe proximal changes, as Crowe type II CHD or more, severe post-surgical modifications such as femoral osteotomy, senile or post-menopausal severe osteoporosis were excluded) in middle-aged patients (range, 55-75 years) make the Mayo stem one of our favorite options in elective adult reconstruction (regularly coupled in the last 3 years with a large diameter femoral head), with a margin of applicability even in intracapsular fractures with at least $15 \%$ of the femoral neck intact (18 cases operated, $11.3 \%$ of total).

Moreover, the essential surgical technique and the straight preparation of the femur make this implant suitable for less and minimally invasive hip approaches, such as MIS-2 [12] and the mini-Watson Jones (as tested in our experience with 19 Mayo stems successfully implanted through a double incision technique and 5 Mayo stems inserted via a mini-anterolateral Watson Jones modified by Bertin and Rottinger [13]). In particular, during MIS-2 technique, even if the entry point of the Mayo stem is quite medial, compared to that of a traditional straight stem, its

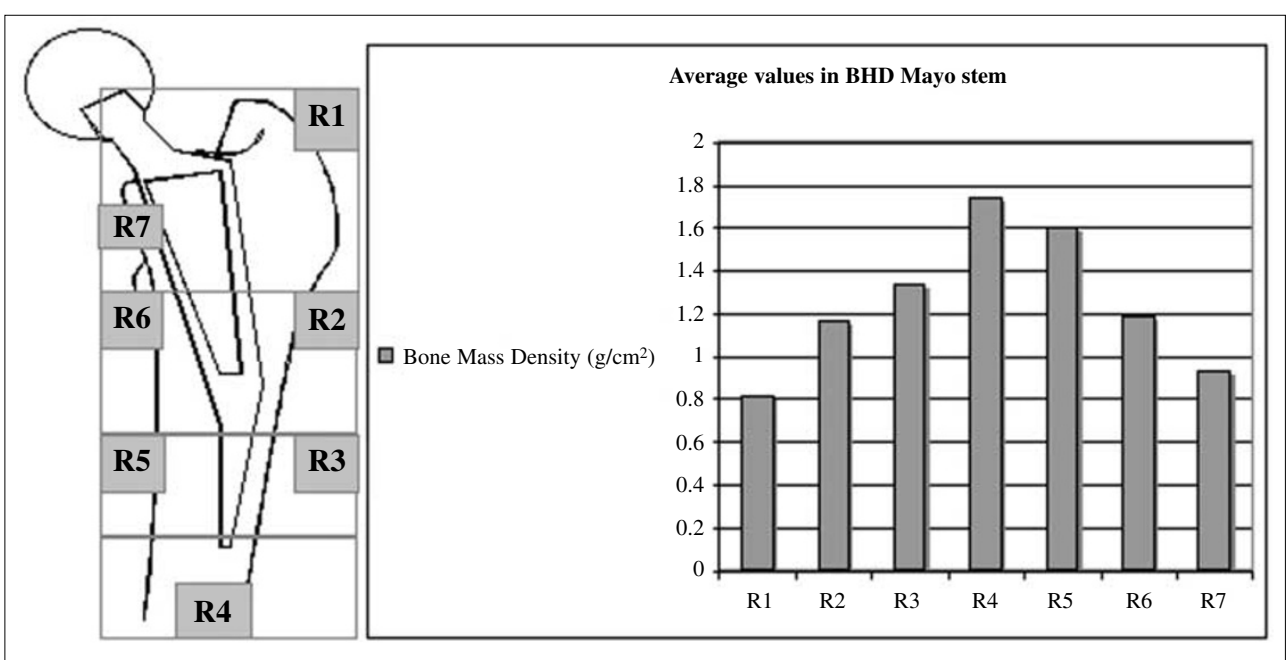

Fig. 6 Prevalence of distal stress transfer along the stem to the lateral femoral cortex 


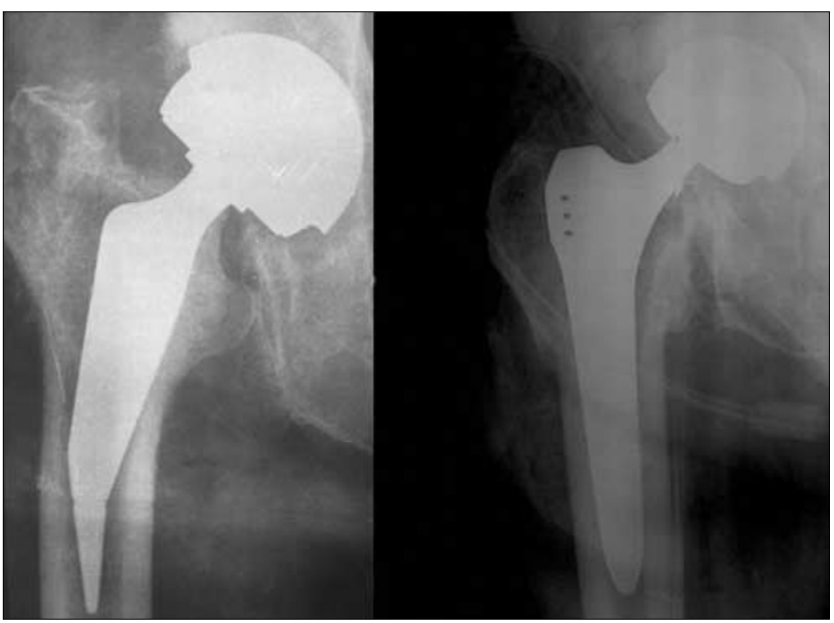

Fig. 7 Revision of a failed Mayo stem with a primary implant

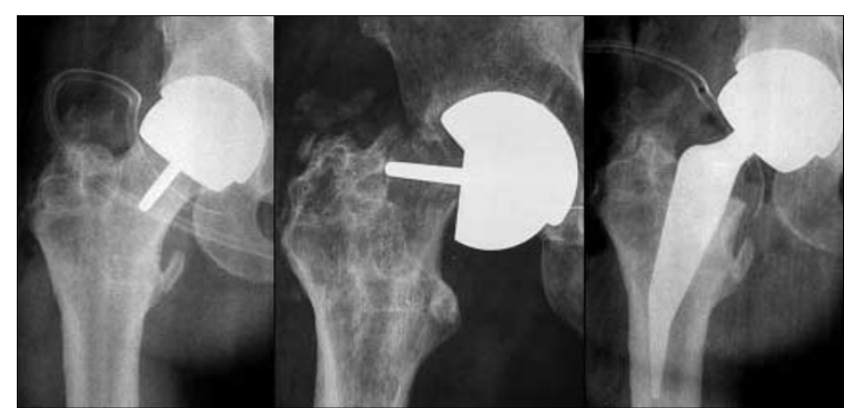

Fig. 8 Revision of a failed resurfacing implant with a Mayo stem

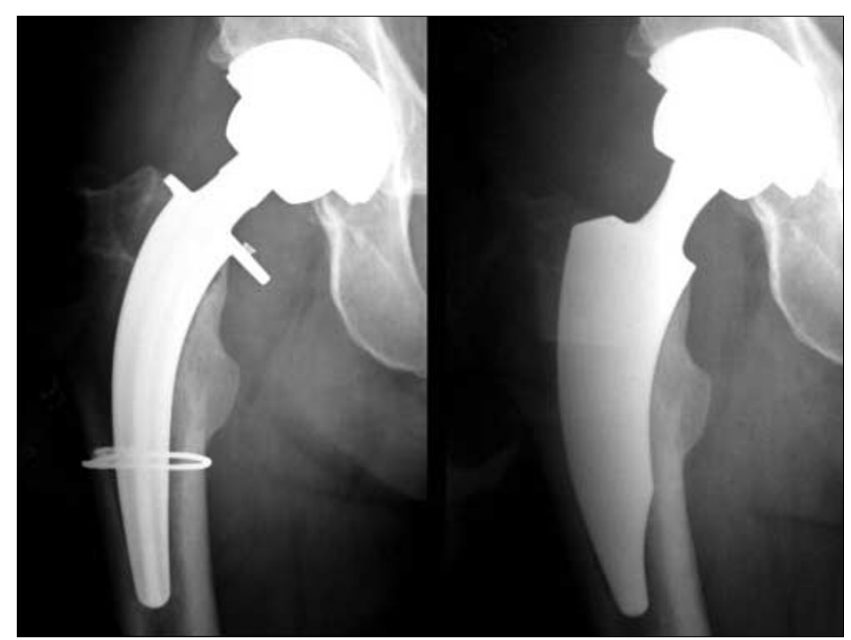

Fig. 9 Revision of a failed CFP stem with a Nanos stem

advance down the canal is definitely straight. After the initial two awls have contacted the lateral cortex, the broaches (and the stem) are impacted sliding along the lateral cortex itself. A similar procedure, which requires only slightly more adduction of the affected leg on to the un-affected side to reach the medial entry point, appears not suitable for other conservative designs, introduced with a curvilinear movement into the metaphysis (such as CFP).
More recently in our experience, the Metha stem seems to offer the same clinical and technical advantages with the addition of femoral neck modularity. Even if majority of the cases can be managed with a standard neck $\left(135^{\circ}\right.$ neckshaft angle, $0^{\circ}$ anteretro-version) in particular situations a modular cervical component may avoid a negative change in bio-mechanic static parameters. Four cases (14.8\%) inour experience with Metha stems were succesfully treated with a non-standard cervical modularity (1 case with $7.5^{\circ}$ of retro-version, 2 hip replaced with $140^{\circ}$ and 1 hip with $130^{\circ}$ of neck-shaft angle). This option may extend the indication to mild proximal femur changes and, retrospectively, would have avoided 4 cases of lateral off-set reduction detected with a Mayo stem (provided a fixed neck-shaft angle). The need for a higher osteotomy, as discussed previously, reduces its application in intra-capsular fractures (only 2 cases in our preliminary experience, $7.4 \%$ of total).

Metaphyseal conservative stems provide undoubted advantages in term of revision. As directly experienced with the Mayo stem, they allow a "conservative revision" in case of failure, with conversion to traditional primary implant (Fig. 7). Moreover, they can represent a conservative option for more conservative failed implants such as resurfacing (revised in our experience with the Mayo stem) (Fig. 8), or CFP (1 case revised in our experience with a Nanos stem (Fig. 9).

\section{Conclusions}

Metaphyseal preserving implants present a biomechanic rationale, a good clinical mid-term behavior and a perspective of easy revision able to place them in the foreground of contemporary hip replacement, even if it is hard to affirm that they may substitute conventional implants or preserving implants with excellent long-term follow-up (such as CFP [1]).

On our advice, they should be elected as the operative solution after more conservative implants have been excluded and a careful balance of epidemiologic (senile and post-menopausal cortical and trabecular bone loss curves) and bio-morphologic parameters (flare index, DEXA analysis) has been performed. A mis-understanding of these parameters may lead, as we unfortunately experienced with both metaphyseal and neck-retaining implants, to rare but dramatic early failures.

\section{References}

1. Pipino F, Keller A (2006) Tissue-sparing surgery: 25 years' experience with femoral neck preserving hip arthroplasty. J Orthop Traumatol 7(1):36-41

2. Carlson L, Albrektsson B, Freeman MA (1988) Femoral neck retention in hip arthroplasty. A cadaver study of mechanical effects. Acta Orthop Scand 59(1):6-8 
3. Pipino F, Calderale PM (1987) Biodynamic total hip prosthesis. Ital J Orthop Traumatol 13(3):289-297

4. Jasty M, Krushell R, Zalenski E, O'Connor D, Sedlacek R, Harris W (1993) The contribution of the nonporous distal stem to the stability of proximally porous-coated canine femoral components. J Arthroplasty 8(1):33-41

5. Whiteside LA, White SE, McCarthy DS (1995) Effect of neck resection on torsional stability of cementless total hip replacement. Am J Orthop (10):766-770

6. Morrey BF (1989) Short-stemmed uncemented femoral component for primary hip arthroplasty. Clin Orthop Relat Res (249):169-175

7. Morrey BF, Adams RA, Kessler M (2000) A conservative femoral replacement for total hip arthroplasty. A prospective study. J Bone Joint Surg Br 82(7):952-958

8. Swanson TV (2005) The tapered press fit total hip arthroplasty: a European alternative. J Arthroplasty 20[4 Suppl 2]:63-67
9. Meldrum RD, Willie BM, Bloebaum RD (2003) An assessment of the biological fixation of a retrieved Mayo femoral component. Iowa Orthop J 23:103-107

10. Albanese CV, Rendine M, De Palama F et al (2006) Bone remodelling in YHA: a comparative DXA scan study between conventional implants and a new stemless femoral component. A preliminary report. Hip Int 16:9-15

11. Roth A, Richartz G, Sander K et al (2005). Periprosthetic bone loss after total hip endoprosthesis. Dependence on the type of prosthesis and preoperative bone configuration. Orthopade 34(4):334-344

12. Berger RA (2004) The technique of minimally invasive total hip arthroplasty using the two-incision approach. Instr Course Lect 53:149-155

13. Bertin KC, Rottiger H (2004) Anterolateral mini-incision hip replacement surgery: a modified Watson-Jones approach. Clin Orthop Relat Res (429):248-255 